\title{
PARÂMETROS BIOLÓGICOS DE BEMISIA TABACI (GENN.) BIÓTIPO B (HEMIPTERA: ALEYRODIDAE) EM GENÓTIPOS DE ALGODOEIRO $\left({ }^{1}\right)$
}

\author{
ZENEIDE RIBEIRO CAMPOS $\left({ }^{2}\right)$; ARLINDO LEAL BOIÇA JÚNIOR $\left({ }^{*}\right)$; \\ ANDRÉ LUIZ LOURENÇÃO $\left(^{3}\right)$; ALCEBÍADES RIBEIRO CAMPOS $\left({ }^{4}\right)$
}

\begin{abstract}
RESUMO
A mosca-branca Bemisia tabaci biótipo B é uma praga de grande importância econômica para muitas culturas em todo o mundo. No Brasil, especialmente no Estado da Bahia, essa praga causou perdas que variam entre $30 \%$ e $70 \%$ em cultura de algodão. Essa pesquisa foi desenvolvida com o objetivo de avaliar parâmetros biológicos de B. tabaci biótipo B em genótipos de algodoeiro para verificar a possível ocorrência de antibiose como mecanismo de resistência sob condições de casa de vegetação. Os genótipos estudados foram IAC-23, Coodetec 406, BRS Aroeira, Fabrika, Coodetec 407, IAC-24, Makina, IAC 20233, Coodetec 401 e CNPA Acala I. Foram avaliados os períodos de incubação e ninfal, o desenvolvimento total e a longevidade. Dos genótipos avaliados observou-se em Coodetec 406 maior período ninfal (14,7 dias) e IAC-23 a menor viabilidade de ninfas $(30,7 \%)$, indicando a ocorrência de resistência do tipo antibiose contra essa mosca-branca.
\end{abstract}

Palavras-chave: mosca-branca, Gossypium hirsutum L., resistência de plantas a insetos, antibiose.

\section{ABSTRACT \\ BIOLOGICAL PARAMETERS OF BEMISIA TABACI B BIOTYPE ON COTTON GENOTYPES}

The silverleaf whitefly Bemisia tabaci B biotype is an important pest of many crops throughout the world. In Brazil, specifically in the Bahia State, this insect caused losses estimated to $30-70 \%$ on cotton crops. The purpose of this research was to investigate biological parameters of the silverleaf whitefly biotype B in genotype of cotton bush to verify a possible occurrence of antibiosis as resistance mechanism cotton, under greenhouse conditions. The duration of the egg and nymphal stages, and the adult longevity of B. tabaci biotype B were evaluated in the genotypes IAC-23, Coodetec 406, BRS Aroeira, Fabrika, Coodetec 407, IAC-24, Makina, IAC 20-233, Coodetec 401 and CNPA Acala I. Among those evaluated genotypes, Coodetec 406 increased the nymph stage (14.7 days) and IAC-23 reduced the nymphal viability $(30.7 \%)$, suggesting the occurrence of antibiosis against this whitefly.

Key words: silverleaf whitefly, Gossypium hirsutum L., host plant resistance, antibiosis.

${ }^{1}$ ) Recebido para publicação em 6 de dezembro de 2007 e aceito em 28 de maio de 2009.

$\left({ }^{2}\right)$ Departamento de Fitossanidade, Faculdade de Ciências Agrárias e Veterinárias/UNESP, Via de Acesso Prof. Paulo Donato Castellane s/n, 14884-900 Jaboticabal (SP). E-mail: aboicajr@fcav.unesp.br (*) Autor correspondente.

${ }^{3}$ ) Instituto Agronômico (IAC), Caixa Postal 28, 13.001-970 Campinas (SP).

( $\left.{ }^{4}\right)$ Depto. de Fitossanidade, Engenharia Rural e Solos, Faculdade de Engenharia/UNESP, Av. Brasil, 56, 15385-000 Ilha Solteira (SP). 


\section{INTRODUÇÃO}

A mosca-branca, Bemisia tabaci (Genn.) se destaca entre as pragas mais nocivas as plantas cultivadas, no mundo, tanto em campo quanto em ambiente protegido (Mound e Halsey, 1978), e seu aumento em importância na agricultura está diretamente relacionado ao aparecimento e à dispersão do biótipo B desta espécie (COSTA e BROWN, 1990). No Brasil, as populações de B. tabaci biótipo B são elevadas nos meses do verão, quando as plantas de algodoeiro estão na fase de abertura das maçãs (SANTOS, 1999).

Os danos diretos causados por ninfas e adultos de $B$. tabaci biótipo $B$ às plantas de algodoeiro são decorrentes de sua alimentação e a injeção de substâncias tóxicas, reduzindo o vigor da planta. Os danos indiretos ocorrem em função da deposição de grande quantidade de seiva sobre folhas e frutos, servindo como substrato para o crescimento de fungos saprófitas (Capnodium sp.), que dificultam a fotossíntese e causam o manchamento de fibras dos capulhos, comprometendo sua qualidade (BLUA e Toscano, 1994; Lourenção e NAGAI, 1994; Chu et al., 2001). A presença de seiva sobre os capulhos causa dano significativo à indústria por dificultar a extração de fibras, danificar as máquinas durante o processamento, alterar a qualidade da fibra e diminuir a lucratividade da cultura (Hector e HodKINSON, 1989; Hequet e Abidi, 2002). Em plantas do algodoeiro infectadas com o vírus do mosaico comum, transmitido pela mosca-branca, pode ocorrer redução na produção em cerca de 50\% (SILVeIRA, 1965).

O controle das moscas-brancas tem sido realizado quase exclusivamente por inseticidas e práticas culturais (VAlle e LouRenção, 2002). Porém, devido às características biológicas e comportamentais de $B$. tabaci biótipo $\mathrm{B}$, como o rápido desenvolvimento, a alta fecundidade e a grande capacidade de dispersão (PRABHAKer et al., 1989; DitTRICH e ERNEST, 1990), o uso freqüente de inseticidas para o controle de altas populações (DARDON, 1993) aumenta a probabilidade de seleção de indivíduos resistentes aos inseticidas reduzindo a eficiência dessa tática de controle (PrabhaKer et al., 1989; Dittrich e ERNEST, 1990; DARDON, 1993).

Em função dos danos causados e devido aos efeitos colaterais provocados pelo uso intensivo de inseticidas, novas alternativas de controle devem ser avaliadas (Horowitz, 1986). Assim, as plantas resistentes representam uma forma importante de controle por reduzir tanto as populações da mosca-branca a níveis subeconômicos (LARA, 1991) e por conseqüência às perdas causadas por esse inseto (McAuslane, 1996).
A presente pesquisa teve por objetivo avaliar parâmetros biológicos de $B$. tabaci biótipo B em genótipos de algodoeiro para verificar a possível ocorrência de antibiose como mecanismo de resistência sob condições de casa de vegetação.

\section{MATERIAL E MÉTODOS}

\section{Criação estoque de B. tabaci biótipo B}

Os adultos de mosca-branca, $B$. tabaci biótipo $B$, utilizados na pesquisa foram adquiridos de colônias mantidas no setor de Entomologia do Instituto Agronômico (IAC), em Campinas. Os insetos foram multiplicados (criação de manutenção) em gaiolas teladas $(2,0 \times 3,0 \times 2,0 \mathrm{~m})$, contendo plantas de couve (Brassica oleraceae var. capitata) e bico-depapagaio (Euphorbia pulcherrima Willd). Quinzenalmente, foram introduzidas plantas novas em substituição às plantas senescentes.

\section{Cultivo dos genótipos}

Plantas dos genótipos IAC-23, Coodetec 406, BRS Aroeira, Fabrika, Coodetec 407, IAC-24, Makina, IAC 20-233, Coodetec 401 e CNPA Acala I foram selecionados com base em trabalho realizado por BoIçA JUNIOR. et al., (2007), que avaliaram a atratividade e a preferência para oviposição de $B$. tabaci biótipo B em vinte genótipos de algodoeiro. As plantas foram cultivadas em vasos de poliestireno, com capacidade para 10 litros. O substrato utilizado foi preparado pela mistura de duas partes de terra, uma parte de areia e uma parte de composto orgânico. A adubação foi feita conforme a recomendação de RAIJ et al. (1997). Na semeadura foram utilizadas quatro sementes por vaso, efetuando-se o desbaste dez dias após a germinação e deixando-se apenas uma planta por vaso. O desenvolvimento das plantas foi acompanhado diariamente, sendo estas irrigadas sempre que necessário. Para o experimento, as plantas foram utilizadas quando estavam com 20 dias após a emergência.

\section{Avaliação de aspectos biológicos de B. tabaci biótipo B}

Inicialmente, foram avaliados o período de incubação e a duração dos estádios ninfais de $B$. tabaci biótipo B nos genótipos de algodoeiro. Utilizou-se o delineamento inteiramente casualizado com seis repetições, e cada planta representou uma repetição. As plantas em experimentação foram mantidas sob telado $(5,0 \times 4,0$ x $3,0 \mathrm{~m}$ ) com temperatura média de $23,8{ }^{\circ} \mathrm{C}$ e umidade relativa média de $70 \%$. 
Utilizaram-se pequenas gaiolas cilíndricas com $4 \mathrm{~cm}$ de diâmetro e $6 \mathrm{~cm}$ de altura, com capacidade para $40 \mathrm{~mL}$, uma por planta dos diferentes genótipos, dentro das quais foram confinados adultos não sexados da mosca-branca. Essas gaiolas foram confeccionadas com copos sem fundo cuja extremidade maior foi fechada com tecido tipo "voile" e a menor protegida com tampa construída com espuma e plástico e fixada por um grampo. Quatro horas após a infestação, as gaiolas foram retiradas, sendo selecionados 50 ovos para avaliação do período de incubação e do desenvolvimento das ninfas.

Durante o período de incubação, foram diariamente contabilizados o número de ovos e o de ninfas recém-eclodidas. Vinte delas, eclodidas no mesmo dia, foram selecionadas para determinação do período ninfal, enquanto as demais foram descartadas. Quando se detectou as primeiras ninfas de quarto estádio (olhos vermelhos), as folhas com ninfas foram individualizadas em gaiolas confeccionadas com caixa plástica $(19 \mathrm{~cm}$ de diâmetro por $8 \mathrm{~cm}$ de altura), protegidas na parte superior com tecido tipo "voile". Cada gaiola contava com uma abertura lateral para permitir a inserção da folha e era fixada ao caule da planta por uma haste de madeira.

Dos adultos recém-emergidos, foram selecionados 10 por repetição para avaliar a longevidade, sem alimentação. Estes insetos foram individualizados em tubos de ensaio transparente $(3,6$ $\mathrm{mL})$ e mantidos em BOD $\left(\mathrm{T}=26 \pm 2^{\circ} \mathrm{C}\right.$; UR $=70 \pm 5 \%$ e fotofase de 12 horas), sem alimentação.

Os dados obtidos para cada variável foram submetidos à análise de variância utilizando-se o software estatístico ESTAT (BARBOSA et al., 1992). Quando significativo, as médias foram comparadas pelo teste de Tukey, a 5\% de probabilidade.

\section{RESULTADOS E DISCUSSÃO}

Com relação ao período médio de incubação de ovos de B. tabaci biótipo B (Tabela 1), a menor média foi observada em Coodetec 407, diferindo de Coodetec 401. Nos outros genótipos, os períodos de incubação variaram de 9,3 a 9,6 dias, sem diferenças significativas entre eles. No geral, os resultados do período de incubação em genótipos de algodoeiro foram intermediários aos relatados por BUTLER JR. et al. (1983), que constataram períodos variando de 5 dias a $32,5{ }^{\circ} \mathrm{C}$ até 22,5 dias a $16,5{ }^{\circ} \mathrm{C}$. Para a mesma cultura, CAmpos (2003) observou médias de 7,5 a 8,7 dias, quando os ovos foram colocados por fêmeas criadas em couve ( $1 .^{a}$ geração), e 8,7 a 12,0 dias, quando os ovos foram colocados por fêmeas criadas em algodoeiro (2. ${ }^{a}$ geração). As porcentagens de viabilidade de ovos foram semelhantes para todos os genótipos, sendo o menor valor detectado em Coodetec 406 (97\%) e nenhuma mortalidade foi observada em Makina (100\%).

Tabela 1. Médias ( \pm EP) do período de incubação e da viabilidade de ovos de B. tabaci biótipo B em dez genótipos de algodoeiro, sob condições de casa de vegetação. Jaboticabal (SP), 2005

\begin{tabular}{lcc}
\hline Genótipos & Período de incubação & Viabilidade \\
\hline Coodetec 401 & $9,9 \pm 0,14 \mathrm{a}$ & $\%$ \\
Makina & $9,6 \pm 0,24 \mathrm{ab}$ & $99,0 \pm 0,68 \mathrm{a}$ \\
Fabrika & $9,5 \pm 0,12 \mathrm{ab}$ & $98,3 \pm 0,00 \mathrm{a}$ \\
CNPA Acala I & $9,5 \pm 0,11 \mathrm{ab}$ & $97,3 \pm 0,84 \mathrm{a}$ \\
IAC-24 & $9,5 \pm 0,06 \mathrm{ab}$ & $99,0 \pm 0,68 \mathrm{a}$ \\
Coodetec 406 & $9,4 \pm 0,10 \mathrm{ab}$ & $97,0 \pm 1,24 \mathrm{a}$ \\
IAC-23 & $9,4 \pm 0,05 \mathrm{ab}$ & $97,3 \pm 1,43 \mathrm{a}$ \\
BRS Aroeira & $9,3 \pm 0,09 \mathrm{ab}$ & $98,0 \pm 1,26 \mathrm{a}$ \\
IAC 20-233 & $9,3 \pm 0,08 \mathrm{ab}$ & $99,7 \pm 0,33 \mathrm{a}$ \\
Coodetec 407 & $9,2 \pm 0,17 \mathrm{~b}$ & $99,0 \pm 0,68 \mathrm{a}$ \\
\hline F & $2,30 *$ & $1,20 \mathrm{~ns}$ \\
Gl (Genótipos) & 9 & 50 \\
Gl (Resíduo) & 9 & 50 \\
CV (\%) & 1,58 & 1,17 \\
\hline
\end{tabular}

Dados originais; para análise estatística foram transformados em $(x+0,5)^{1 / 2}$; Médias seguidas da mesma letra não diferem entre si pelo teste de Tukey $(\mathrm{p}=0,05)$.

As menores médias de duração do período ninfal foram observados em IAC 23 e Makina (Tabela 2), diferindo de Coodetec 406 que exigiu maior tempo de desenvolvimento. Períodos ninfais de 13,5 a 14,3 dias foram observados para os outros genótipos, os quais não diferiram dos valores extremos 13,3 e 14,7 respectivamente. CAMPOS (2003) reporta que os genótipos Fibermax 986, BRS Antares e FMT possuem características que conferem resistência por antibiose a B. tabaci biótipo B, evidenciada pelo maior período de tempo gasto para completar o desenvolvimento ninfal desta praga. A menor viabilidade ninfal foi verificada em IAC-23 (30,7\%), que diferiu de Coodetec $406(64,2 \%)$, Coodetec 407 (63,7\%) e BRS Aroeira (53,3\%). Em trabalho semelhante a cultivar EPAMIG Alva, avaliada por CAmpos (2003), revelou viabilidade de $8 \%$ de ninfas de B. tabaci biótipo B, indicando a presença de fatores antibióticos que atuaram de forma efetiva no controle do inseto. Para os outros genótipos, a viabilidade variou entre $39,3 \%$ e $45,7 \%$, não diferindo dos valores extremos, observados em Coodetec 407 (63,7\%), BRS Aroeira $(53,3 \%)$ e IAC $23(30,7 \%)$. 
Tabela 2. Médias ( \pm EP) do período de ninfal, do desenvolvimento de ovo a adulto e viabilidade ninfal de $B$. tabaci biótipo B em dez genótipos de algodoeiro, sob condições de casa de vegetação. Jaboticabal (SP), 2005

\begin{tabular}{lccc}
\hline Genótipos & Período ninfal & Viabilidade & Desenvolvimento de ovo a adulto \\
\hline Coodetec 401 & dias & $\%$ & dias \\
Makina & $13,5 \pm 0,29 \mathrm{ab}$ & $45,0 \pm 4,70 \mathrm{ab}$ & $23,4 \pm 0,29 \mathrm{ab}$ \\
Fabrika & $13,3 \pm 0,37 \mathrm{~b}$ & $41,3 \pm 3,29 \mathrm{ab}$ & $22,9 \pm 0,25 \mathrm{ab}$ \\
CNPA Acala I & $13,6 \pm 0,31 \mathrm{ab}$ & $45,7 \pm 3,52 \mathrm{ab}$ & $23,1 \pm 0,26 \mathrm{ab}$ \\
IAC-24 & $13,7 \pm 0,20 \mathrm{ab}$ & $40,3 \pm 2,09 \mathrm{ab}$ & $23,2 \pm 0,26 \mathrm{ab}$ \\
Coodetec 406 & $13,6 \pm 0,28 \mathrm{ab}$ & $39,3 \pm 1,84 \mathrm{ab}$ & $23,1 \pm 0,27 \mathrm{ab}$ \\
IAC-23 & $14,7 \pm 0,32 \mathrm{a}$ & $64,2 \pm 8,79 \mathrm{a}$ & $24,1 \pm 0,31 \mathrm{a}$ \\
BRS Aroeira & $13,3 \pm 0,25 \mathrm{~b}$ & $30,7 \pm 3,45 \mathrm{~b}$ & $22,7 \pm 0,27 \mathrm{~b}$ \\
IAC 20-233 & $14,0 \pm 0,23 \mathrm{ab}$ & $53,3 \pm 7,65 \mathrm{a}$ & $23,3 \pm 0,31 \mathrm{ab}$ \\
Coodetec 407 & $14,0 \pm 0,18 \mathrm{ab}$ & $45,7 \pm 9,19 \mathrm{ab}$ & $23,3 \pm 0,23 \mathrm{ab}$ \\
F & $14,3 \pm 0,29 \mathrm{ab}$ & $63,7 \pm 7,35 \mathrm{a}$ & $23,4 \pm 0,21 \mathrm{ab}$ \\
Gl (Genótipos) & $2,50^{*}$ & $4,25^{*}$ & $1,78^{\mathrm{ns}}$ \\
Gl (Resíduo) & 9 & 9 & 9 \\
CV (\%) & 50 & 50 & 50
\end{tabular}

Dados originais; para análise estatística foram transformados em $(x+0,5)^{1 / 2}$; Médias seguidas da mesma letra não diferem entre si pelo teste de Tukey $(p=0,05)$.

A duração de ovo a adulto de B. tabaci biótipo $B$ foi influenciada de forma significativa pelos diferentes genótipos (Tabela 2). O menor período, com 22,7 dias, foi verificado no genótipo IAC 23 diferindo de Coodetec 406, com 24,1 dias, o qual causou aumento no ciclo do inseto. Resultados verificados por TORRES et al. (2007), em algodoeiro, revelam variação, não significativa, entre 19,7 e 20,8 dias para os períodos de ovo a adulto de $B$. tabaci biótipo B em oito cultivares de algodoeiro avaliadas.

Nenhuma diferença foi observada quanto à longevidade de adultos de B. tabaci biótipo B, provenientes de genótipos de algodoeiro (Figura 1). Em valores absolutos, indivíduos sem alimentação sobreviveram por menor tempo nos genótipos Coodetec 401 e Makina (1,9 dias) e, quando se considerou todos os genótipos, a média geral foi de 2,09 dias. Indiferentemente do que foi observado nesta pesquisa, TsaI e WANG (1996) asseguram que a longevidade de $B$. tabaci biótipo B é afetada pela planta hospedeira e a disponibilidade de alimento. Os resultados constatados por CAMPOS (2003) são concordantes com esta afirmativa à medida que a longevidade de adultos de B. tabaci biótipo B, alimentados em genótipos de algodoeiro, foi significativamente diferente. O tempo de vida de adultos da mosca-branca foi significativamente maior no genótipo FMT Saturno comparativamente a BRS Itaúba, Louisiana Okra-2, Makina e Epamig Alva.

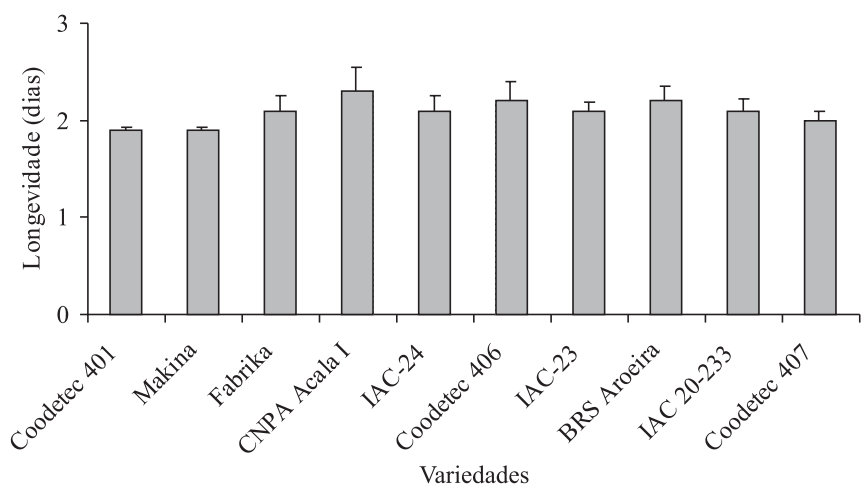

Figura 1. Longevidade de adultos de B. tabaci biótipo B em genótipos de algodoeiro, sob condições de casa de vegetação. Jaboticabal (SP), 2005.

Com base nesses resultados, verifica-se que os genótipos Coodetec 406 e IAC-23 contêm fatores que conferem resistência por antibiose a $B$. tabaci biótipo $B$, caracterizadas por induzir maior duração do período ninfal (14,7 dias) para Coodetec 406 e menor viabilidade de ninfas $(30,7 \%)$ para IAC-23.

\section{REFERÊNCIAS}

BARBOSA, J.C.; MALHEIROS, E.B.; BANZATTO, D.A. ESTAT: um sistema de análises estatísticas de ensaios agronômicos. Jaboticabal: Unesp, 1992. Versão 2.0. 
BLUA, M.J.; TOSCANO, N.C. Bemisia argentifolii (Homoptera: Aleyrodidae) development and honeydew production as a function of cotton nitrogen status. Environmental Entomology, v.23, p.316-321, 1994.

BOIÇA JUNIOR, A.L.; CAMPOS, Z.R.; LOURENÇÃO, A.L.; CAMPOS, A.R. Adult attractiveness and oviposition preference of Bemisia tabaci (Genn.) (Homoptera: Aleyrodidae) B-biotype in cotton genotypes. Scientia Agricola, v.64, p. 147-151, 2007.

BUTLER JR., G.D.; HENNEBERRY, T.J.; CLAYTON, T.E. Bemisia tabaci (Homoptera: Aleyrodidae): development, oviposition, and longevity in relation to temperature. Annals of the Entomological Society of America, v.76, p.310-313, 1983.

CAMPOS, O.R. Resistência de genótipos de algodoeiro a mosca branca Bemisia tabaci (Gennadius 1889) biótipo B (Hemiptera: Aleyrodidae). 2003. 69f. Tese (Doutorado em Agronomia) Faculdade de Ciências Agronômica, Universidade Estadual Paulista, Botucatu.

CHU, C.C.; FREEMAN, T.P.; BUCKNER, J.S.; HENNEBERRY, T.J.; NELSON, D.R.; NATWICK, E. Susceptibility of upland cotton cultivars to Bemisia tabaci biotype B (Homoptera: Aleyrodidae) in relation to leaf age and trichome density. Annals of the Entomological Society of America, v.94, p.743749, 2001.

COSTA, H.S.; BROWN, J.K. Variability in biological characteristics, isozyme patterns and virus transmission among populations of Bemisia tabaci in Arizona. Phytopathology, v.80, p.888, 1990.

DARDON, S.D. Las moscas blancas en Guatemala. In: Las moscas blancas (Homoptera: Aleyrodidae) en America Cental y El Caribe. Catie, 1993. p.38-41. (Informe Técnico nº 205)

DITTRICH, V.S.; ERNST, G.H. Chemical control and insecticide resistance of whiteflies. In: GERLING, D. Whiteflies: their bionomics, pest status and management. Hampshire, England, 1990. p.263-284.

HECTOR, D.J.; HODKINSON, I.D. Stickiness in cotton. Oxon: CAB International, 1989, 43p.

HEQUET, E.; ABIDI, N. Processing sticky cotton: implication of trehalulose in residue build-up. Journal of Cotton Science, v.6, p.77-90, 2002.

HOROWITZ, A.R. Population dynamics of Bemisia tabaci (Gennadius): with special emphasis on cotton field. Agriculture Ecosystems and Environment, v.17, p.37-47, 1986.

LARA, F.M. Princípios de resistência de plantas a insetos. São Paulo: Ícone, 1991. 336p.

LOURENÇÃO, A.L.; NAGAI, H.1994. Surtos populacionais de Bemisia tabaci no Estado de São Paulo. Bragantia, v.53, p.53-59, 1994.

McAUSLANE, H.J. Influence of leaf pubescence on ovipositional preference of Bemisia argentifolii (Homoptera: Aleyrodidae) on soybean. Environmental Entomology, v.25, p.834-41, 1996.
MOUND, L.A; HALSEY, S.M. Whitefly of the world. British Museum (Natural History), London: John Wiley, 1978. 340p.

PRABHAKER, N.; TOSCANO, N.C.; COUDRIET, D.L. Susceptibility of the immature and adult stages of the sweetpotato whitefly (Homoptera: Aleyrodidae) to selected inseticides. Journal of Economic Entomology, v.82, p.983988, 1989.

RAIJ, B. V.; CANTARELLA, H.; QUAGGIO, J. A.; FURLANI, A. M. C. Recomendações de adubação e calagem para o Estado de São Paulo. Campinas: IAC/Fundação IAC, 1997. 285p. (Boletim Técnico, 100)

SANTOS, W.J. Monitoramento e controle das pragas do algodoeiro. In: CIA, E. FREIRE, E.C.; SANTOS, W. J. Cultura do algodoeiro. Piracicaba: Potafós, 1999. p. 179.

SILVEIRA, A.P. Moléstias. Fungos e bactérias. In: NEVES, O. da S.; CAVALERI, P.A.; VERDADE, F. da C.; JUNQUEIRA, A.A.B.; GRIDI-PAPP, I.L.; ORTOLANI, A.A.; SILVA, N.M. da; RIGHI, N.R.; FERRAZ, C.A.M.; CORREAA, D.M.; CALCAGNOLO, G.; SILVEIRA, A.P.; COSTA, A.S.; CARVALHO, A.M.B.; MENDES, H.C.; FUZATTO, M.G.; CORRÊA, F.; BERZAGHI, M.N. Cultura e adubação do algodoeiro. São Paulo: Instituto Brasileiro de Potassa, 1965. p.417-33.

TORRES, L.C.; SOUZA, B; AMARAL, B.B.; TANQUE, R.L. Biology and non-preference for oviposition by Bemisia tabaci (Gennadius) biotype B (Hemiptera: Aleyrodidae) on cotton cultivars. Neotropical Entomology, v.36, p.445-453, 2007.

TSAI, J.H.; WANG, K. Development and reproduction of Bemisia argentifolii (Homoptera: Aleyrodidae) on five host plants. Environmental Entomology, v.25, p.810-816, 1996.

VALLE, G.E.; LOURENÇÃO, A.L. Resistência de genótipos de soja a Bemisia tabaci (Genn.) biótipo B (Hemiptera: Aleyrodidae). Neotropical Entomology, v.31, p.285-295, 2002. 